\title{
VOLATILE PROFILE AND CONSUMER ACCEPTABILITY OF NATURAL YOGHURTS ELABORATED WITH CHILEAN NATIVE CULTURES OF ENTEROCOCCUS SP. STRAIN BB3 AND LACTOBACILLUS SP. STRAIN BB6
}

\author{
SHARON VISCARDI ${ }^{1,5,6^{*}}$, LUIS MARILEO ${ }^{2,6}$, MARIA PAZ ARAOS $^{1}$, JAVIERA STANDEN $^{1}$, SILVANA CERNA ${ }^{1}$, \\ MARIO AMATO ${ }^{3}$ AND CRISTIAN PAZ ${ }^{4 *}$ \\ ${ }^{l}$ Laboratorio de investigación en Salud de Precisión, Departamento de Procesos Diagnóstico y Evaluación, Facultad de Ciencias de la Salud, Universidad \\ Católica de Teтисо, Manuel Montt 56, Campus San Francisco -Temuco, Chile. \\ ${ }^{2}$ Programa de Doctorado en Ciencias Agropecuarias, Facultad de Recursos Naturales, Universidad Católica de Temuco, Chile. Rudecindo Ortega 02950, \\ Temuco, La Araucanía, Chile. \\ ${ }_{3}^{3}$ Political Sciences Department, Universitá degli Studi di Napoli Federico II, Via Rodinó 22/a, 80138, Napoli, Italia. \\ ${ }^{4}$ Departamento de Ciencias Básicas, Universidad de La Frontera, Francisco Salazar Av. 1145-Temuco, Chile. \\ ${ }^{5}$ Núcleo de investigación en Producción Alimentaria, Universidad Católica de Temuco, Rudecindo Ortega 02950 - Temuco, Chile. \\ ${ }^{6}$ Biotechnology of Functional Foods Laboratory, Camino Sanquilco, Parcela 18, Padre Las Casas, La Araucanía, Chile.
}

\begin{abstract}
According to WHO, in 2016 around $13 \%$ of the world's adult population (11\% of men and $15 \%$ of women) were obese. The global prevalence of obesity almost tripled between 1975 and 2016. In this context, it is important to note that the continuous increase in life expectancy, the desire for a better quality of life, and the high cost of medical care have led to a growing demand for functional foods in the market. People have started to realize that their food choices and their unhealthy lifestyles have consequences for their health. An important role in this scenario is associated with functional foods containing probiotic strains, reported to benefit human health. Here we report the development of natural yogurts based on native probiotic starter cultures of Enterococcus sp. strain BB3 (MK681869) and Lactobacillus sp. strain BB6 (MK681868), isolated from raw cow milk of La Araucanía region of Chile. The odor and taste were the main parameters criticized by the consumer, the analysis of CG-MS elucidated that the yogurt produced by the strain Enterococcus sp. strain BB3 (MK681869) produced a higher amount of heptan2 -one, associated with a cheesy type odor, with less acceptance by the consumers. The acceptability of 6 types of yogurt was evaluated with a randomized analysis of 60 participants. The development of a functional dairy product based on probiotics is of interest because of its relation with positive changes in gut microbiota, and at the same time with a possible decrease in body weight.
\end{abstract}

Keywords: Functional Probiotic Yogurt, Autochthonous Starter cultures, Lactic Acid Bacteria, Volatile Compounds, CG-MS.

\section{INTRODUCTION}

A Functional Food is defined as a food able to benefit one or more functions of the human organism through its nutritional effects, thus improving the state of health, reducing the risk of some diseases, and/or counteracting minor physiological disorders ${ }^{1,2}$. One category of Functional Foods whose importance is increasingly recognized is probiotics-based food. Probiotics are defined as living microorganisms that, administered in adequate amounts, may benefit human health ${ }^{3}$. These microorganisms shape aspects of the host's metabolism and immune function, and also regulate numerous aspects of human physiology, including the elimination of exogenous toxins, the regulation of intestinal functions, and the synthesis, absorption and metabolism of nutrients ${ }^{4}$.

It is also reported that obesity is related to gut microbiota through mechanisms such as increment of energy extraction from food, production of fat deposit, and effects on satiety and systemic inflammation ${ }^{5}$. De Vos \& de Vos (2012) reported the association between obesity and gut microbiota to be the inverse of the Bacteroides/Firmicutes ratio, and showed that this inverse ratio is higher with weight $\operatorname{loss}^{6}$. The gut microbiota of healthy individuals is composed of $90 \%$ Firmicutes or Bacteroides cells, which are very sensitive to changes in diet, since a diet high in fats and sugar increases the relative abundance of Firmicutes, contributing to weight gain ${ }^{7,8}$.

According to WHO, the global prevalence of obesity almost tripled between 1975 and 2016. On the other hand, people have started to realize that their food choices and their unhealthy lifestyles have consequences for their health ${ }^{9}$. Renewed interest in fermented foods and probiotics has been observed in recent years, driven mainly by their benefits for human health ${ }^{10}$. Probiotics, mainly Lactic Acid Bacteria (LAB), have been incorporated in a wide range of foods especially dairy products - due to their benefits for both the gastrointestinal tract and bodyweight control ${ }^{11,12}$. Yogurts were produced using starter cultures of Streptococcus thermophilus and Lactobacillus delbrueckii subsp. bulgaricus have been the most sold in the USA and other countries ${ }^{13}$.

Based on the relationship established between probiotics, gut microbiota, obesity, and increasing consumer consciousness, it is clear that probiotics strains are helpful in the production of functional yogurt. The development of natural yogurts based on native probiotic starter cultures, with good acceptance of consumers without the use of sweeteners, could be a policy to explore in obesity prevention.

\section{EXPERIMENTAL}

\subsection{Isolation, characterization, and selection of LAB strains}

Raw fresh milk was obtained from Agroforestal y Ganadería Hector Damasio Antillanca Vejar of Ciudad de la Paz, Loncoche in La Araucanía Region of Chile $\left(39^{\circ} 22^{\prime} 00^{\prime \prime} \mathrm{S} 72^{\circ} 38^{\prime} 00^{\prime \prime} \mathrm{W}\right)$ and used to isolate putative LAB strains as described by Wang et al. $(2016)^{14}$. Genomic DNA from the putative LAB strains ${ }^{15}$ was extracted using a DNeasy UltraClean Microbial Kit (QIAGEN, Hilden, Germany). Partial 16S rRNA genes were amplified as described by Peace et al. $(1994)^{16}$. The PCR products were purified and sent to Macrogen, Inc. (Seoul, Korea) for Sanger sequencing. The $16 \mathrm{~S}$ rRNA gene microbial reference database NCBI was used for taxonomic identification of the putative LAB isolates at genus level (> 90\% identity). The 16S rRNA gene sequences identified in the study were deposited in GenBank and aligned using Clustal Omega.

\subsection{Yogurt preparation}

Milk was fermented by incubating the selected strains of LAB in $900 \mathrm{ml}$ of pasteurized full-cream milk at a concentration of $1 \%(\mathrm{v} / \mathrm{v})$ for 18-24 hours at 20$25^{\circ} \mathrm{C}^{17,18}$. The test strains were incubated alone or in combination (consortium).

\subsection{Sensory descriptive analysis}

Six different yogurt combinations were used to study sensorial properties (Table 1): EN (Enterococcus sp. strain BB3-based yogurt), ES (Enterococcus sp. strain BB3-based yogurt in combination with stevia at a concentration of $0.3 \%$ ), ET (Enterococcus sp. strain BB3-based yogurt in combination with tagatose at a concentration of 1.3\%), LN (Lactobacillus sp. strain BB6-based yogurt), LS (Lactobacillus sp. strain BB6-based yogurt in combination with stevia at a concentration of $0.3 \%$ ), LT (Lactobacillus sp. strain BB6-based yogurt in combination with tagatose at a concentration of $1.3 \%$ ).

The level of acceptance of the fermented product was evaluated through sensory analysis by an untrained group of adult people between 18 and 50 years old $(n=60)$, chosen as judges, under two selection criteria: time availability and no aversion to yogurt ${ }^{19}$.

The descriptors chosen for the quantitative descriptive analysis of the different yogurt samples were eight parameterss that included visual objective, gustative/tactile and olfactory evaluation. 
The parameterss considered were: texture, acidity, sweetness, olfactory intensity and color, rated on 7-point hedonic scales from (1) "Strongly disagree" to (7) "Strongly agree" (7). The result was a unique result for each product ${ }^{20}$. The judges were requested to drink water prior to and during the tasting to reduce any residual effect.

Table 1. Yogurt combinations used to study sensorial properties.

\begin{tabular}{|l|c|c|c|}
\hline Strain/ Sweater & No Sweetner & Tagatose 1.3\% & Stevia 0.3\% \\
\hline Lactobacillus sp. BB6 & LN & LT & LS \\
\hline Enterococcus sp. BB3 & EN & ET & ES \\
\hline
\end{tabular}

\subsection{Extraction and CG-MS analysis of volatile compounds}

Twenty $\mathrm{mL}$ of water and $15 \mathrm{ml}$ of dichloromethane $(\mathrm{CH} 2 \mathrm{Cl} 2)$ were added to $10 \mathrm{~mL}$ of yogurt, then the mixture was extracted 3-Folds and the organic layers were pooled together and dried with anhydrous $\mathrm{MgSO} 4$. The solvent was softly removed in vacuum at $500 \mathrm{mmHg}$ and $30^{\circ} \mathrm{C}$ on a Büchi R100 rotavap. The extract was diluted until $2 \mathrm{mg} / \mathrm{mL}$ with $\mathrm{CH} 2 \mathrm{Cl} 2$, and the sample was kept frozen at $-80^{\circ} \mathrm{C}$ until its analysis.

The volatile compounds were identified by gas chromatography mass spectrometry (CG-MS) on an Agilent 7890 and Agilent 5975 mass detector, California, USA, using a column capillary of silica HP5-MS of $30 \mathrm{~m} \times 0.25 \mathrm{~mm}$ and a film of $0.25 \mu \mathrm{m}$ in thickness. Mass spectra were obtained from total ion current (TIC) and compared to standard mass spectra in addition to the NIST05a database, National Institute of Standards and Technology, NIST 2018.

\subsection{Statistical analysis of results}

Principal Component Analysis (PCA, PRIMER 6 version 6.1.16, PRIMER-e Ltda. 2013) was used to study attribute-sample relationships. The PCA created a sensory attribute-sample space in which samples were positioned according to their characteristic sensory attributes. Significant differences were determined by one-way ANOVA; the threshold of significance was set at $\mathrm{p}<0.05$.

\section{RESULTS AND DISCUSSION}

Two strains of Lactic acid bacteria were isolated from raw milk produced in La Araucanía region (Chile). They were identified as Enterococcus sp. strain BB3 and Lactobacillus sp. strain BB6, and deposited in GenBank under the accession numbers MK681869 and MK681868 respectively. As both strains are reported in literature as probiotics, as shown in the phylogenetic tree (Figure 1), they were selected as starter cultures for fermentation in UHT full-cream milk until a creamy, consistent yogurt was obtained.

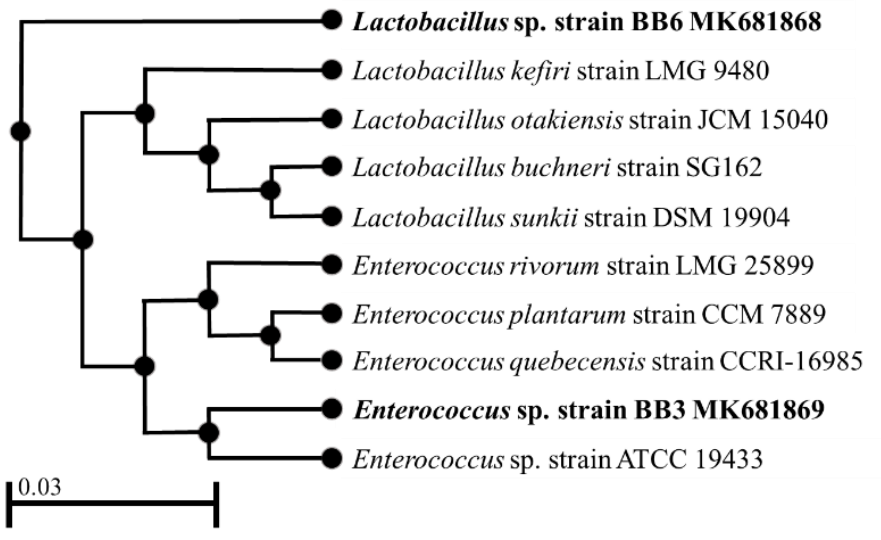

Figure 1. Phylogenetic distance tree displaying the high similarity between probiotic strains existent in Genebank database and Enterococcus sp. BB3 MK681869 and Lactobacillus sp. BB6 MK681868 strains isolated in this study.

Three conditions were defined: 1) Lactobacillus sp. strain BB6; 2) Enterococcus sp. strain BB3; 3) combination of the two strains. Strains were preinoculated at $1 \%$ in sterile skimmed milk at $37^{\circ} \mathrm{C}$ for 24 hours, achieving a concentration of $1 \times 10^{7} \mathrm{UFC} / \mathrm{ml}$. The pre-inoculum was used as a starter inoculum to begin fermentation of the milk. Three liters of UHT full-cream milk, one for each of the conditions described above, were inoculated under sterile conditions with the three combinations of the selected strains at $1 \%$, achieving a final concentration of $1 \times 10^{6} \mathrm{UFC} / \mathrm{ml}$.

The combination of the two strains did not work as starter culture, as it did not reach the desired characteristics.

Sensory evaluation was performed with the help of 60 untrained judges using a hedonic scale. Organoleptic differences between the two yogurts are shown in Figure 2. Note that the Lactobacillus sp. strain BB6-based yogurt obtained greater acceptance for its texture; on the other hand, Enterococcus sp. strain BB3based yogurt appears to be sweeter. The acidity of both products was evaluated at an average of 6 on the scale. Both yogurts were evaluated by the judges in combination with the sweeteners stevia and tagatose. Surprisingly, in the case of Enterococcus sp. strain BB3-based yogurt there were no significant differences in the sweetness parameters between natural yogurt and yogurt with added sweetener; the product itself presented a pleasant sweetness for the tasters, therefore the addition of a sweetener is unnecessary.

Principal component analysis was performed to identify patterns in the attribute-sample relationship from the judges' perceptions. The PCA indicates that the highest percentage of total variance is found between $\mathrm{PC} 1$ and $\mathrm{PC} 2$, with a cumulative variance of $74.5 \%$. The coefficients in the linear combinations of the variables that make up the PCs indicate that the variables olfactory intensity (OI) and acidity (Ac) are attributed to PC1 with coefficients of 0.717 and 0.568 respectively. The perception of the consumers presents a positive correlation with the EN, ES, ET, LS and LT samples for the attributes texture (Te), color (Co) and sweetness ( $\mathrm{Sw})$; for the particular case of $\mathrm{LN}$ the analysis presents a positive correlation with the attributes olfactory intensity and acidity. Measurement of the resemblance by the Bray Curtis similarity index indicated high similarity between all the samples of $92 \%$, and only $8 \%$ differentiation between the samples EN, ES, ET, LS and LT against LN; these differences are attributed mainly to the attributes $\mathrm{OI}$ and $\mathrm{Ac}$ in the $\mathrm{LN}$ sample.

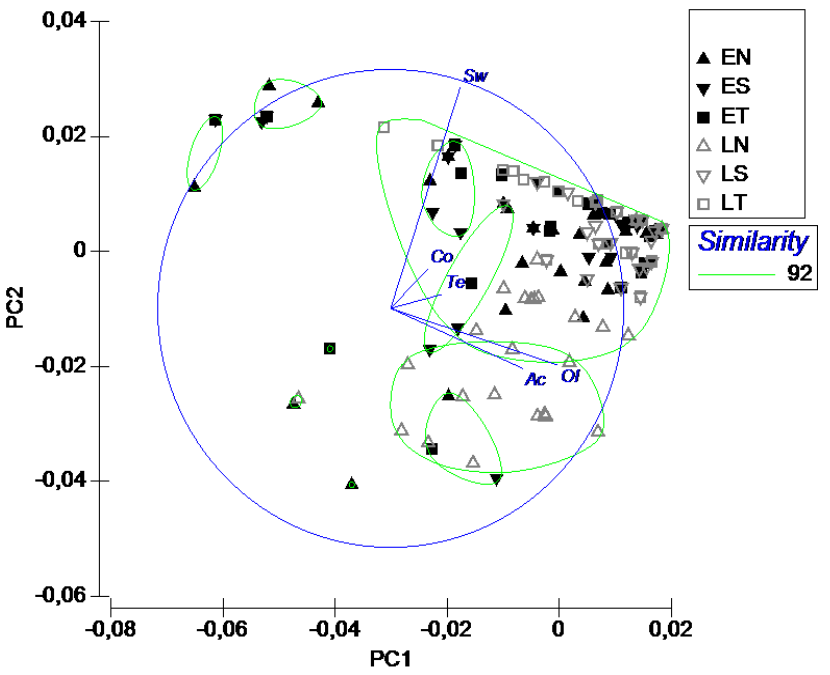

Figure 2. Principal component analysis (PCA) based on descriptive sensory attributes (texture, acid, sweet, olfactive intensity, texture and color) of six yogurt combinations: EN (Enterococcus sp. BB3 strain based yogurt), ES (Enterococcus sp. BB3 strain based yogurt in combination with Stevia at the concentration of 0.3\%), ET (Enterococcus sp. BB3 strain based yogurt in combination with tagatose at the concentration of 1.3\%), LN (Lactobacillus sp. BB6 strain based yogurt), LS (Lactobacillus sp. BB6 strain based yogurt in combination with Stevia at the concentration of 0.3\%), LT (Lactobacillus sp. BB6 strain based yogurt in combination with tagatose at the concentration of $1.3 \%)$.

The volatile compounds were analyzed by CGMS analysis. Similar pattern was observed for the yogurt produced by Lactobacillus sp. and the yogurt produced by Enterococcus sp. (Figure 3). The main compounds identified by the NIST05a database are summarized in the Table 2, they are represented for small molecules previously identified in lactic fermentations related to yogurt and/or cheese process $^{21,22}$. These molecules given in the table 2 have characteristic smells associated with fermented fatty acids, buttery and acidic, which are not considered unpleasant for the people. 


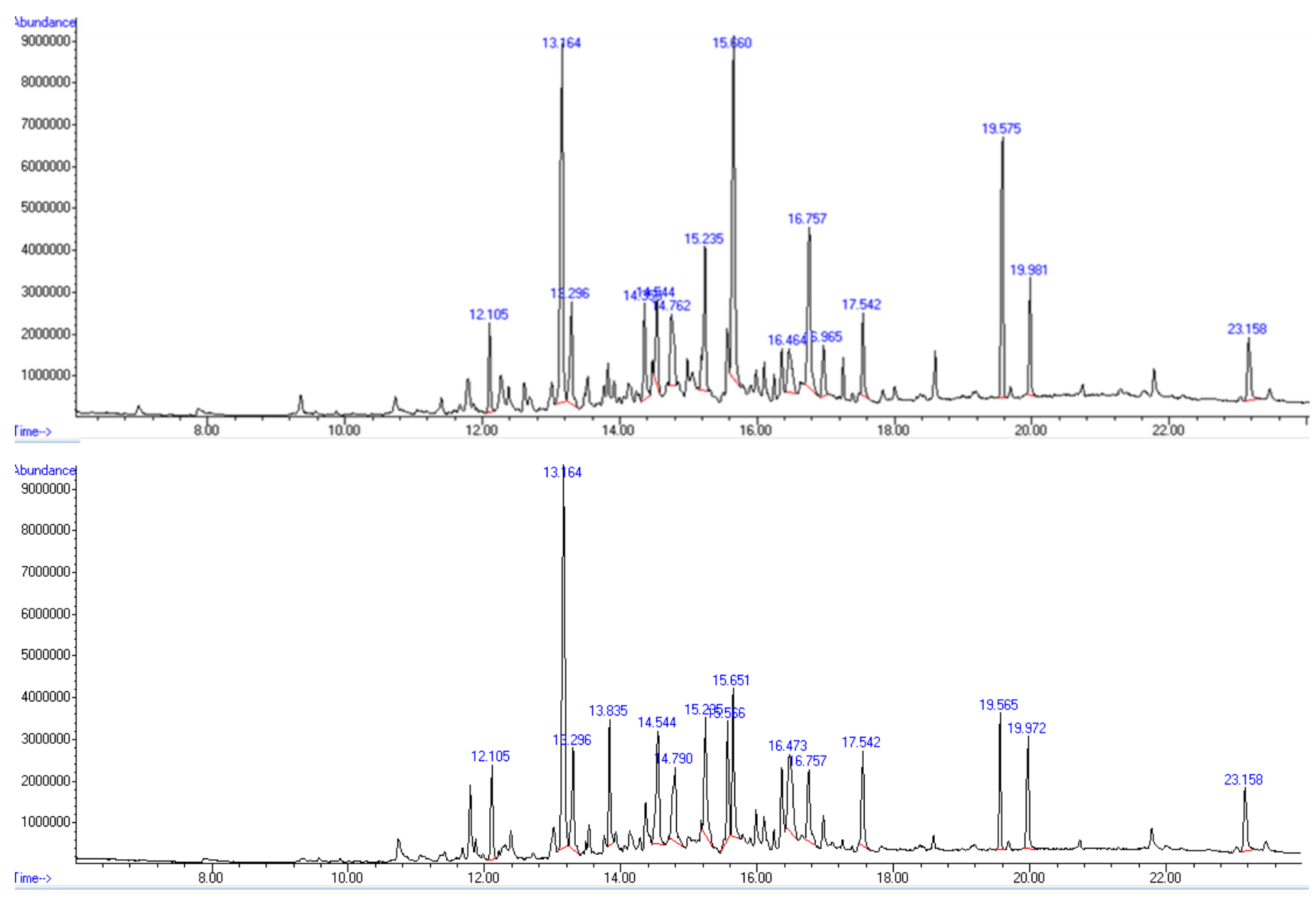

Figure 3. Representative CG-MS of yogurt based on Enterococcus sp. BB3 strain (A) and Lactobacillus sp BB6 strain (B), time in minutes.<smiles>CCCCOCCCCC(C)=O</smiles><smiles>CCCCOC(=O)CC(CC(=O)OCCCC)(OC(C)=O)C(=O)OCCCC</smiles>

Heptan-2-one

3-methylbutan-1-ol

Figure 4. Structure of volatile compounds of yoghurt by CG-MS.

Table 2. Odor profile of volatile compounds of yogurt by CG-MS

\begin{tabular}{|c|l|c|c|l|}
\hline Retention time (min) & \multicolumn{1}{|c|}{ Compound } & Lactobacillus sp. BB6 & Enterococcus sp. BB3 & Descriptors \\
\hline 12.105 & 2,3-pentanedione & $\mathrm{xx}$ & $\mathrm{xx}$ & Buttery, caramellic \\
\hline 13.164 & Butan-1-ol & $\mathrm{xxxx}$ & $\mathrm{xxxx}$ & Buttery, pungent \\
\hline 15.660 & Heptan-2-one & $\mathrm{x}$ & $\mathrm{xxxx}$ & cheese type odour \\
\hline 16.757 & 3-methylbutan-1-ol & $\mathrm{x}$ & $\mathrm{xx}$ & fermented type odour \\
\hline 17.542 & Tributyl acetylcitrate & $\mathrm{x}$ & $\mathrm{x}$ & slightly viscous liquid with very faint sweet herbaceous odour \\
\hline 19.575 & Diisooctyl phthalate & $\mathrm{xx}$ & $\mathrm{xxx}$ & No information \\
\hline 19.981 & Lactic acid ethyl ester & $\mathrm{xx}$ & $\mathrm{xx}$ & buttery, creamy \\
\hline 23.158 & Acetic acid & $\mathrm{x}$ & - & Sour, vinegar-like, pungent \\
\hline
\end{tabular}

Number of $\mathrm{x}$ is given as a relative abundance from less $\mathrm{x}$ to higher $\mathrm{xxxx}$. 
The gut microbiota plays an important role in the absorption, storage, and waste of energy obtained from the $\operatorname{diet}^{5}$, and it is also involved in the regulation of food intake by affecting the hormones that influence the metabolism, function and areas in the brain which are associated with eating behavior. This microbial axis, called brain-gut axis, represents a bidirectional relationship, and regulated body weight through appetite balance, storage and energy waste ${ }^{23}$. In this sense, obesity and malnutrition are alterations in the composition and diversity of the gut microbiota, which are in opposite with healthy individuals ${ }^{7,8}$. This alteration in gut microbiota is known as dysbiosis, and it is associated with altered body weight and fat storage ${ }^{24}$. Thus, it is clear that gut microbiota plays an important role in energy homeostasis, glucose metabolism and the metabolism of host lipids, therefore the use of probiotics is a strategy to prevent and to treat obesity or overweight through a wide variety of anti-obesogenic mechanisms such as: modulation of energy metabolism, improvement in the intestinal barrier, reduction of low-grade inflammation, modulation of autonomic nervous activity, and modulation of the appetite ${ }^{25}$.

Lactic acid bacteria are commonly found in the human gastrointestinal tract and represent the majority of probiotics present in the market that performs the activity of exclusion or inhibition of pathogens, enhancing intestinal epithelial barrier and improving the capacity of degradation of non-digestible food components ${ }^{3}$.

In this work, two strains of lactic acid bacteria were isolated from raw fresh cow's milk. These strains were identified and Enterococcus sp. BB3 and Lactobacillus sp. BB6, both genres are reported as probiotics. It has been reported that the Enterococcus sp. strain can benefit both the food industry and health, since it has had the role of starter culture in several food industries ${ }^{26}$ ) and it has shown an anti-obesity effect in mice induced with a high-fat diet, which would imply the possibility of attenuating hepatic steatosis by its administration $^{27}$.

On the other hand, a study carried out by Kim et al. $(2017)^{25}$, showed that Lactobacillus sp. inhibited the development of $60 \%$ of obesity induced by high fat diets, manifested by the decrease in adiposity and weight gain. In addition, it is emphasized that the strain seemed to have a double function, cholesterol reduction in vitro and acceleration of fatty acid oxidation in adipose tissue.

A tasting of natural yogurt made of probiotics was carried out to an untrained group of 60 people belonging to Universidad Católica de Temuco. The results have established the favorable acceptance to the fermented products (Figure 2), maybe because the yogurts were produced with Lactobacillus sp. and with Enterococcus sp. have a creamy texture, good flavor even without sweeteners the smell was not unpleasant. Aromatic compounds in yogurt are came from lipolysis of milkfat and microbiological transformations of aldehydes and fatty acids, producing more than 100 volatile compounds related to small organic compounds as alcohols, acids, esters or hydrocarbons, including: acetaldehyde, 2-butanone, lactic acid, acetoin, acetone ${ }^{21}$. Here we found characteristic molecules founded in yogurts as 2,3-pentanedione, butan-1-ol, heptan-2-one, 3methylbutan-1-ol, tributyl acetylcitrate, lactic acid ethyl ester and acetic acid, other compounds were not well correlated to the chemical library, for that reason its study require a deeper chemical work. The yogurt produced by Lactobacillus sp. had a stronger smell than the yogurt produced by Enterococcus sp. this difference is possible to see in the CGMS analysis (Figure 4 and Figure 5), which have a similar pattern but not the same, for instance the Enterococcus sp. produced higher content of heptan-2-one which is associated with a strong smell to cheesy type odor, also the consistency of this yogurt turns to more viscous with time. Small molecules and less polar compounds previously reported ${ }^{21,22}$ were not visible. Anyway, this is a preliminary potential of both bacteria present in a local milk, which could be useful in the industry.

This is a relevant result since yogurt and fermented milk are among the most common fresh dairy products consumed around the world ${ }^{28}$, having great relevance with gastrointestinal health and more specifically with a contribution to the gut microbiota ${ }^{29}$. It is important to notice that Chile is the country with the highest annual consumption of yogurt per person in South America with approximately 14 liters per person (ODEPA, 2016).

An interesting aspect of natural fermented yogurt based on LAB fermentation is the content of water-soluble essential vitamins. Particularly the amount of folate (vitamin B9) may be increased to values above $200 \mu \mathrm{g} / \mathrm{L}$ depending on the starter cultures used and the storage condition, which can contribute to satisfying the recommended dietary allowance ( $400 \mu \mathrm{g}$ DFE), highlighting the plus value of such type of fermented food ${ }^{30,31}$.
However, despite the fact that the authorities recommend their consumption as part of a balanced diet, two out of three consumers say to add sugar, marmalade or honey, and thus increasing its caloric density and decreasing the benefits of the product ${ }^{28}$. The reduction of the sugar content in foods is a great issue of interest to control dietary intake and reduce obesity rates ${ }^{32}$. The rapid increase in the prevalence of obesity worldwide has been partially attributed to the excessive consumption of added sugars; in this context, the current recommendation to limit added sugars in the diet can be replaced by caloric and non-caloric sweeteners ${ }^{33}$.

\section{CONCLUDING REMARKS}

Aroma and flavor are ones of the most important properties determining the acceptability and preference of functional fermented milks ${ }^{21,34}$. The Study of consumers' acceptance of functional yogurts in Temuco (La Araucanía Region, Chile) indicated that if the consumer has a job or has had access to information on functional foods, increased majority acceptance of enriched functional yogurt ${ }^{35}$. In this research work we achieved a well-accepted natural functional yogurts were produced by autochthonous probiotics strains of Lactobacillus sp. BB\& and Enterococcus sp. BB3 strains isolated from raw cow milk of La Aracanía Region of Chile.

In particular, the yogurts were well accepted in terms of color, texture, aroma, acidity and sweetness.

We consider that this could be a preliminary study of consumer attitudes in Chile towards a natural fermented yogurt based on LAB which, consumable without sugar, could be marketed as a strategy to promote healthy lifestyle and nutrition.

\section{ACKNOWLEDGMENTS}

This research was supported by Project number VIP-UCT-412-4447, funded by Universidad Católica de Temuco, Chile.

\section{REFERENCES}

1. I. Jankovic, W. Sybesma, P. Phothirath, E. Ananta, A. Mercenier, Curr. Opin Biotechnol. 21, 175, (2010).

2. M.F. Chen, Appetite. 57, 253, (2011)

3. M. H. Floch, Gastroenterol. Clin. North Am. 47, 179, (2018)

4. M. L. Battson, D. M. Lee, T. L. Weir, C. L. Gentile, J. Nutr. Biochem. 56, 1, (2018).

5. F. Tsai, W. J. Coyle, Curr. Gastroenterol. Rep. 11, 307, (2009)

6. W. M. de Vos, E. A. de Vos, Nutr. Rev. 70, S45, (2012).

7. P. J. Turnbaugh, F. Bäckhed, L. Fulton, J. I. Gordon, Cell Host Microbe. 3 , 213, (2008).

8. E. D. Sonnenburg, H. Zheng, P. Joglekar, S. K. Higginbottom, S. J. Firbank, D. N. Bolam, J. L. Sonnenburg, Cell. 141, 1241, (2010).

9. B. Goetzke, S. Nitzko, A. Spiller, Appetite. 77, 96, (2014).

10. F. Melini, V. Melini, F. Luziatelli, A. G. Ficca, M. Ruzzi, Nutrients. 11, 1189 , (2019).

11. J. Burgain, C. Gaiani, M. Linder, J. Scher, J. Food Eng. 104, 467, (2011).

12. A. Astrup, Am. J. Clin. Nutr. 99, 1235S, (2014).

13. E. W. Ng, M. Yeung, P. S. Tong, Int. J. Food Microbiol. 145, 169, (2011).

14. D. Wang, W. Liu, Y. Ren, L. De, D. Zhang, Y. Yang, Q. Bao, H. Zhang, B Menghe, Korean J. Food Sci. Anim. Resour. 36, 499, (2016).

15. A. J. Rondón, L. M. Samaniego, R. Bocourt, S. Rodríguez, G. Milián, M. J. Ranilla, M. Laurencio, M. Pérez, Cienc. y Tecnol. Aliment. 6, 56, (2008).

16. T. A. Peace, K. V. Brock, H. F. Stills, Int. J. Syst. Bacteriol. 44, 832, (1994),

17. B. Nielsen, G. C. Gürakan, G. Ünlü, Probiotics Antimicrob. Proteins. 6, 123 (2014).

18. A. Machado, D. O. Leite, M. Antonio, L. Miguel, R. S. Peixoto, A. S. Rosado, J. T. Silva, V. Margaret, F. Paschoalin, Brazilian J. Microbiol. 349, 341, (2013).

19. I. Acevedo Pons, O. García, J. Contreras, I. Acevedo, Rev. Cient. UDO Agric 9, 442, (2009).

20. C. Catania, S. Avagnina, Curso Super. degustación vinos, 26, (2007).

21. H. Cheng, Crit. Rev. Food Sci. Nutr. 50, 938, (2010).

22. A. Ott, L. B. Fay, A. Chaintreau, J. Agric. Food Chem. 45, 850, (1997).

23. S. M. O'Mahony, G. Clarke, Y. E. Borre, T. G. Dinan, J. F. Cryan, Behav. Brain Res. 277, 32, (2015)

24. N. C. de Clercq, A. K. Groen, J. A. Romijn, M. Nieuwdorp, Adv. Nutr. An Int. Rev. J. 7, 1080, (2016).

25. D.-H. Kim, D. Jeong, I.-B. Kang, H. Kim, K.-Y. Song, K.-H. Seo, Mol. Nutr Food Res. 61, 1700252, (2017) 
26. A. K. Mishra, A. R. Ghosh, Probiotics Antimicrob. Proteins. 10, 435, (2018).

27. M. Kondoh, T. Shimada, K. Fukada, M. Morita, K. Katada, Y. Higashimura, K. Mizushima, M. Okamori, Y. Naito, T. Yoshikawa, Br. J. Nutr. 112, 868, (2014).

28. A. Saint-Eve, H. Leclercq, S. Berthelo, B. Saulnier, W. Oettgen, J. Delarue, Appetite. 99, 277, (2016).

29. M. L. Marco, D. Heeney, S. Binda, C. J. Cifelli, P. D. Cotter, B. Foligné, M. Gänzle, R. Kort, G. Pasin, A. Pihlanto, E. J. Smid, R. Hutkins, Curr. Opin. Biotechnol. 44, 94, (2017).

30. J. L. Revuelta, C. Serrano-Amatriain, R. Ledesma-Amaro, A. Jiménez, Appl. Microbiol. Biotechnol. 102, 8613, (2018).
31. S. Viscardi, L. Marileo, P. J. Barra, P. Durán, C. Inostroza-Blancheteau, Curr. Opin. Food Sci. 34, 1, (2020).

32. C. H. Edwards, M. Rossi, C. P. Corpe, P. J. Butterworth, P. R. Ellis, Trends Food Sci. Technol. 56, 158, (2016).

33. A. D. Mooradian, M. Smith, M. Tokuda, Clin. Nutr. ESPEN. 18, 1, (2017).

34. D. D. Pan, Z. Wu, T. Peng, X. Q. Zeng, H. Li, J. Dairy Sci. 97, 624, (2014).

35. B. Schnettler, C. Shene, M. Rubilar, H. Miranda, J. Sepúlveda, M. Denegri, G. Lobos, Arch. Latinoam. Nutr. 60, 380, (2010). 